\title{
Roles and Impacts of the Transplant Pharmacist: A Systematic Review
}

\author{
Sébastien Sam, Aurélie Guérin, André Rieutord, Stéphanie Belaiche, and Jean-François Bussières
}

\begin{abstract}
Background: Pharmacists have been involved in the care of transplant recipients for several decades, and a growing body of literature shows the beneficial effects of clinical pharmacist care on important outcomes for these patients.
\end{abstract}

Objectives: The primary objective was to describe the roles and impacts of pharmacists in a solid organ transplant setting. The secondary objective was to describe and rate the pharmacists' interventions.

Data Sources: Three databases - PubMed, Embase, and Evidence-Based Medicine Reviews —-were searched from January 1, 1990, to June 16, 2015.

Study Selection and Data Extraction: All studies addressing the roles of pharmacists and the impacts of clinical pharmacy services on the care of solid organ transplant recipients were considered. Only studies providing a statistical analysis were included. Design, setting, sample size, patient characteristics, pharmacists' interventions, study bias, and outcomes were extracted for analysis.

Data Synthesis: Four randomized controlled trials, 4 cohort studies, 3 pre-post studies, and 1 quasi-randomized controlled trial were included in the review, representing a total of 1837 patients. Of the 12 studies included, 8 specifically focused on renal transplant, and 1 each focused on liver, lung, abdominal organ, and general solid organ transplant. The pivotal pharmacist activities leading to the main patient outcomes were medication counselling ( $n=8$ studies), medication reconciliation $(n=5)$, and reviewing and optimizing drug therapy $(n=3)$. Improvements to medication adherence ( $n=6$ studies), morbidity $(n=4)$, costs $(n=2)$, and medication errors $(n=2)$ were reported.

Conclusion: Currently available evidence suggests that pharmacists can improve patient outcomes in the solid organ transplant setting. Adherence, morbidity, costs, and medication errors were identified as the main outcomes that were improved by pharmaceutical interventions. Transplant programs need to invest more in this resource.

Keywords: pharmacist, organ transplantation, impact, clinical pharmacy, outcome-based research

Can J Hosp Pharm. 2018;71(5):324-37

\section{RÉSUMÉ}

Contexte : Les pharmaciens participent aux soins des greffés depuis plusieurs décennies et un nombre croissant de publications révèlent les effets bénéfiques des soins prodigués par les pharmaciens cliniciens quant aux résultats thérapeutiques importants pour ces patients.

Objectifs : L'objectif principal était de décrire les rôles des pharmaciens et leurs influences par rapport aux greffes d'organes solides. L'objectif secondaire était de décrire et d'évaluer les interventions des pharmaciens.

Sources des données : Les bases de données PubMed, Embase et Evidence-Based Medicine Reviews ont été interrogées pour la période

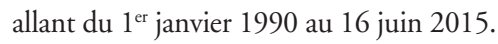

Sélection des études et extraction des données : Toutes les études abordant les rôles des pharmaciens et l'influence des services de pharmacie clinique sur les soins des receveurs d'organes solides ont été prises en considération. Seules les études présentant des analyses statistiques ont été retenues. Le plan d'étude, le contexte, la taille de l'échantillon, les caractéristiques des patients, les interventions des pharmaciens, les biais et les résultats thérapeutiques ont servi à l'analyse.

Synthèse des données : Quatre études contrôlées à répartition aléatoire, 4 études de cohorte, 3 études avant-après et 1 essai comparatif à répartition quasi-aléatoire ont été retenus pour l'analyse, ce qui représentait au total 1837 patients. Parmi les 12 études retenues, 8 abordaient spécifiquement la greffe rénale et chacune des 4 autres concernait respectivement une greffe hépatique, une greffe pulmonaire, une greffe d'organe abdominal et une greffe d'organe solide. Les activités clés des pharmaciens menant aux principaux résultats thérapeutiques étaient les conseils sur les médicaments ( $n=8$ études), l'établissement du bilan comparatif des médicaments $(n=5)$ ainsi que l'examen et l'optimisation de la pharmacothérapie $(n=3)$. On a constaté des améliorations des taux d'observance pharmacothérapeutique ( $n=6$ études), des taux de morbidité $(n=4)$, des coûts $(n=2)$ et des taux d'erreurs de médicaments $(n=2)$.

Conclusion : Les données probantes disponibles laissent croire que les pharmaciens peuvent améliorer les résultats thérapeutiques en ce qui concerne les greffes d'organes solides. Les taux d'observance pharmacothérapeutique, les taux de morbidité, les coûts et les taux d'erreurs de médicaments ont été désignés comme les résultats principaux qui ont été améliorés par les interventions pharmaceutiques. Les programmes de greffe doivent investir davantage dans cette ressource.

Mots clés : pharmacien, greffe d'organe, effet, pharmacie clinique, recherche axée sur les résultats 


\section{INTRODUCTION}

Colid organ transplant has been one of the most important$\checkmark$ therapeutic advances in medicine over the past 60 years. Since the first transplants were performed, it has become the recommended therapeutic approach for many end-stage chronic diseases. In Canada, 2835 transplant procedures were done in $2016 .^{1}$

Patients who have received a solid organ transplant require lifelong immunosuppressive treatments. Nonadherence to posttransplant drug therapy and recommendations is a major issue that can lead to misdiagnosis of subsequent health problems, poor health affecting quality of life, graft rejection, or death., ${ }^{2,3}$

Pharmacists have been involved in direct patient care since the early 1970s. The first report outlining specific activities of a dedicated transplant pharmacist was published in $1976 .{ }^{4}$ This article introduced the transplant pharmacist as an individual with specific expertise in transplantation pharmacology who actively participated in the medical management of organ transplant recipients and provided direct patient medication counselling. Since that time, the overall pharmacy practice model has evolved from a product-oriented to a patientoriented model, and there have been advances in the field of transplant pharmacy as well. In the United States, for example, a "pharmacology expert" is now mandatory in transplant centres. $^{5}$

A growing body of literature has shown the beneficial effects of clinical pharmacist care on important outcomes for both hospitalized and ambulatory patients; however, in the context of solid organ transplant, the majority of published studies have focused on renal transplant recipients.

There is high heterogeneity among the interventions described in studies evaluating the impact of clinical pharmacy services. Several authors have characterized the descriptions of interventions in pharmacy practice studies as inconsistent or even poor. ${ }^{6,7}$ Authors have therefore recommended that interventions be clearly reported, with a detailed explanation of the intervention, a description of the pharmacist-patient and pharmacist-provider relationships, and details about the setting where the study took place. ${ }^{8}$ A more comprehensive understanding of clinical pharmacy interventions for transplant patients would help in achieving better outcomes.

The primary objective of this systematic review was to describe the roles and impacts of pharmacists in a solid organ transplant setting. The secondary objectives were to describe and rate pharmacists' interventions.

\section{METHODS}

All specifications of the PRISMA 2009 checklist $^{9}$ were followed for reporting this systematic review.

\section{Data Sources}

Four systematic searches were carried out in 3 databases (PubMed, Embase, and Evidence-Based Medicine Reviews) for articles published between January 1, 1990, and June 16, 2015. Manual reference checks were performed to search for potentially missing studies. Search strategies are presented in Appendix 1 (available at https://www.cjhp-online.ca/ index.php/cjhp/issue/view/186/showToc).

\section{Study Selection and Data Extraction}

All studies addressing the impact of clinical pharmacy services on the care of patients with solid organ transplant were considered. Studies providing a statistical analysis on the impact of pharmaceutical activities were included. Studies that presented only descriptive results, studies addressing only the economic impact of transplant services, descriptive reviews, case reports, journal letters, journal notes, commentaries, and editorials were all excluded. Also excluded were secondary sources such as literature reviews, systematic reviews, and metaanalyses. Articles in either English or French were included.

All references were screened by 2 independents reviewers (A.G., J.F.B.). If there were any discrepancies in the decision to include or exclude studies, a third researcher was consulted (S.B.). Study selection was accomplished through 3 phases of screening. During the first phase, titles were reviewed for relevance. During the second phase, abstracts from articles retained in the first phase were reviewed for relevance. In the third and final phase, the full texts of articles retained in the second phase were reviewed.

Data extraction was performed by 2 authors (A.G., S.S.), under the supervision of 1 reviewer (J.F.B.). Data from the included studies were synthesized into summary tables.

\section{Rating of Descriptions of Pharmaceutical Interventions}

The DEPICT tool ${ }^{10}$ was used to evaluate the description of pharmaceutical interventions. Rating was performed by 2 authors (A.G., S.S.), under the supervision of 1 reviewer (J.F.B.), and a DEPICT score was assigned to each study. The DEPICT score evaluates studies according to 12 sections, with multiple items per section. For each section, a score of 1 is assigned if the reviewers answer "yes" for at least 1 item within the section; otherwise, a score of 0 is assigned for that section. The DEPICT score is determined by summing the number of sections with a score of 1 (maximum score $=12$ ).

\section{Risk of Bias in Individual Studies}

Individual study limitations, including risk of bias, were reported as described by the authors of each included article. The risk of bias across studies was assessed informally by the authors of the current systematic review. 


\section{RESULTS}

\section{Literature Search, Study Selection, and Data Extraction}

The search yielded 1603 articles. Of these, 1518 were excluded after review of titles and abstracts. Of the 85 potentially eligible studies, 73 were excluded after review of the full-text articles. Twelve studies involving a total of 1837 patients were included in the analysis (Figure 1). ${ }^{1-22}$ Manual searching of the reference lists of these included articles yielded no additional eligible articles.

\section{Synthesis of Results}

Eight studies focused on kidney transplant, one on liver transplant, one on lung transplant, one on abdominal transplant, and one on general solid organ transplant. The studies were conducted in the United States ( $n=8$ studies), Canada $(n=2)$, and Germany $(n=2)$. No differences were observed in terms of pharmacist roles or patient outcomes in relation to the geographic location of the studies.

The study characteristics are presented in Table 1 and the outcomes of individual studies in Table 2.

The pivotal pharmacist activities in the setting of solid organ transplant included patient education and counselling ( $n=9$ studies), reviewing and optimizing drug therapy $(n=7)$, and medication reconciliation or medical history $(n=5)$. Improvements were reported in the following areas: medication adherence ( $n=6$ studies), morbidity $(n=4)$, cost $(n=2)$, and medication errors $(n=2)$.

Pharmaceutical interventions were sufficiently described to understand the role of pharmacists. The average DEPICT score was 8.4 (standard deviation 1.4 , minimum 6, maximum 11) (Table 3). The pharmaceutical interventions that were less frequently reported included the timing of the intervention, the support resources provided by pharmacists, and the pharmacist's autonomy to perform some specific tasks.

\section{Risk of Bias}

Risk of bias is reported here as described by the authors of each article (Table 1). Many studies lacked a control group and had a small sample size. Three of the included studies were carried out by the same multidisciplinary renal transplant team at the Medical College of Georgia Hospital and Clinics. ${ }^{15,19,21}$ A fourth study had the same first author as these 3 studies (Marie A Chisholm-Burns, formerly Marie A Chisholm), but was conducted within a different organization. ${ }^{17}$

\section{DISCUSSION}

Our detailed literature search identified few studies describing the inclusion of clinical pharmacists as members of multidisciplinary teams in the organ transplant setting. In these studies, transplant pharmacists were involved in medication reconciliation, drug therapy evaluation and monitoring, patient education, and problem-solving. All of the studies included in our review suggested that transplant pharmacists could improve the management and medication adherence of patients and consequently could have a positive impact on patients' morbidity, medication errors, and costs. However, each of the studies was conducted in a single centre, and it might be difficult to show significant evidence of a pharmacist's impact in small, focused patient populations like these.

The number of studies that met our inclusion criteria ( $n=12$ ) was low compared with studies examining the roles of pharmacists in other settings (e.g., cancer, hypertension, and asthma). ${ }^{23}$ In fact, the involvement of clinical pharmacists in transplant medicine is recent. The American Society of HealthSystem Pharmacists now offers a pharmacy residency in solid organ transplant, ${ }^{24}$ but no European recommendations have been formulated regarding the role of the clinical pharmacist in transplantation. Lack of knowledge and/or experience in designing and administering such services, as well as difficulty in procuring funding and reimbursement for services, can limit

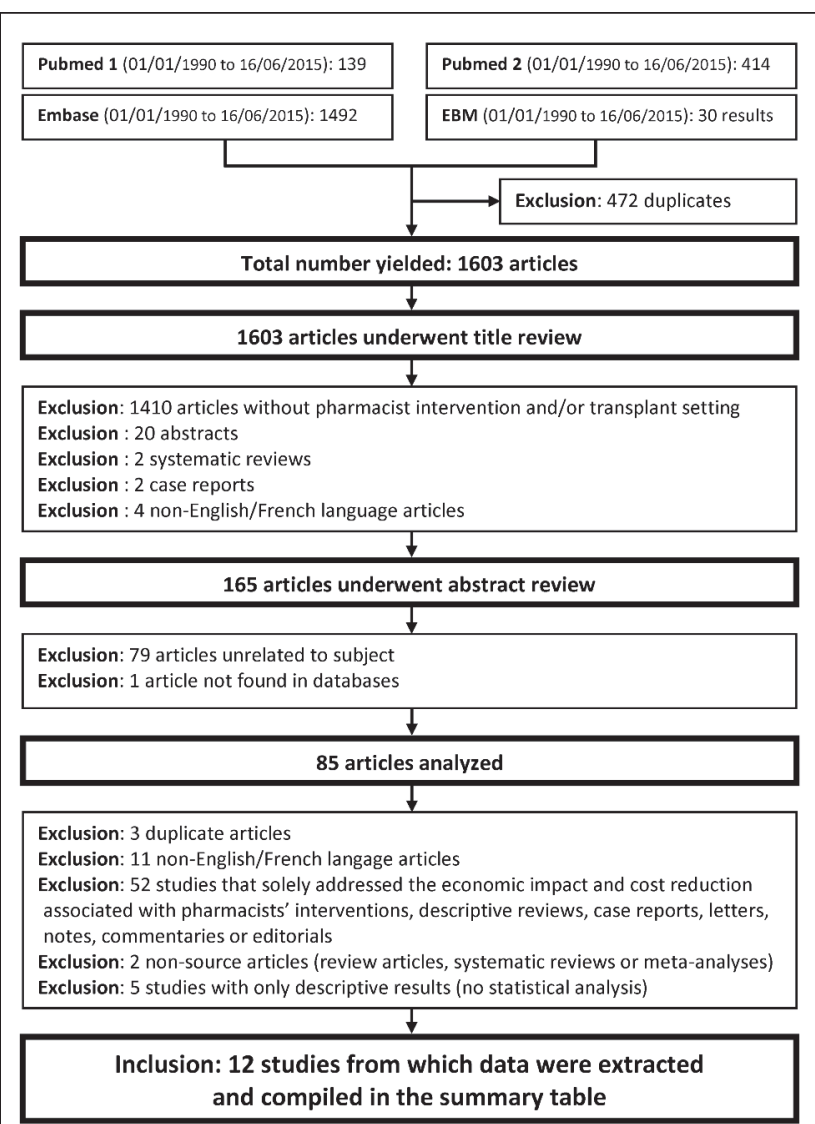

Figure 1. Selection of studies for a systematic review of the roles and impacts of transplant pharmacists. 
Table 1 (part 1 of 4). Study Characteristics

\begin{tabular}{|c|c|c|c|c|c|}
\hline Reference & $\begin{array}{l}\text { Study Design } \\
\text { and Timeframe }\end{array}$ & Setting & $\begin{array}{c}\text { Sample Size } \\
\text { and Patient } \\
\text { Characteristics }\end{array}$ & $\begin{array}{l}\text { Pharmacists' } \\
\text { Interventions }\end{array}$ & Bias \\
\hline \multicolumn{6}{|c|}{ Randomized controlled trials } \\
\hline $\begin{array}{l}\text { Chisholm et al. } \\
2001^{15}\end{array}$ & $\begin{array}{l}\text { RCT, prospective } \\
\text { February } 1997 \text { to } \\
\text { January } 1999\end{array}$ & $\begin{array}{l}\text { United States: } \\
\text { Medical College of } \\
\text { Georgia-Hospital } \\
\text { and Clinics } \\
\text { Renal transplant } \\
\text { clinic }\end{array}$ & $\begin{array}{l}\text { Control }(C): n=12 \\
\text { Intervention (I): } \\
n=12 \\
\text { Mean age } \pm \text { SD: } \\
49.2 \pm 10.2 \text { years } \\
\text { Sex, male: } \\
75 \%(18 / 24) \\
\text { Kidney transplant } \\
\text { recipients }\end{array}$ & $\begin{array}{l}\text { At least monthly direct } \\
\text { patient care clinical services } \\
\text { in person or by phone: } \\
\text { - Obtaining medication } \\
\text { histories } \\
\text { - Reviewing and optimizing } \\
\text { medication therapy } \\
\text { - Making recommendations } \\
\text { to the nephrologists } \\
\text { - Providing oral and/or written } \\
\text { medication counselling for } \\
\text { patients }\end{array}$ & $\begin{array}{l}\text { - To strengthen compliance } \\
\text { assessment, serum drug } \\
\text { concentrations were } \\
\text { measured, but patients may } \\
\text { have increased compliance } \\
\text { before the blood samples and } \\
\text { may have been inaccurate } \\
\text { due to incorrect sampling } \\
\text { times in relation to } \\
\text { medication administration } \\
\text { - Small sample size }(n=24)\end{array}$ \\
\hline $\begin{array}{l}\text { Chisholm et al. } \\
2002^{21}\end{array}$ & $\begin{array}{l}\text { RCT, prospective } \\
\text { Inclusion from } \\
\text { November } 1996 \text { to } \\
\text { March } 1998\end{array}$ & $\begin{array}{l}\text { United States: } \\
\text { Medical College } \\
\text { of Georgia } \\
\text { Renal transplant } \\
\text { clinic }\end{array}$ & $\begin{array}{l}\text { Control }(\mathrm{C}): n=10 \\
\text { Intervention }(\mathrm{I}) \text { : } \\
n=13 \\
\text { Mean age } \pm \text { SD } \\
\mathrm{C}: 47 \pm 12.7 \text { years } \\
\mathrm{I}: 51 \pm 16.8 \text { years } \\
\text { Sex, male: } \\
\mathrm{C}: 70 \% \\
\mathrm{I}: 61.5 \% \\
\text { African-American } \\
\text { kidney recipients }\end{array}$ & $\begin{array}{l}\text { Direct care clinical pharmacy } \\
\text { services: } \\
\text { - Meeting with patient at } \\
\text { least twice monthly during } \\
\text { the first } 3 \text { months after } \\
\text { transplant, at least monthly } \\
\text { during months 4-8, and } \\
\text { at least once during } \\
\text { months 8-12 } \\
\text { - Giving information about } \\
\text { the medication } \\
\text { - Obtaining medication } \\
\text { histories } \\
\text { - Reviewing medication } \\
\text { therapy, with emphasis on } \\
\text { controlling blood pressure } \\
\text { - Preventing or resolving } \\
\text { medication problems } \\
\text { - Sending recommendations } \\
\text { to the nephrologists }\end{array}$ & $\begin{array}{l}\text { - Contamination bias: } \\
\text { members of health care team } \\
\text { may have progressively been } \\
\text { influenced by the } \\
\text { pharmacist's } \\
\text { recommendations, affecting } \\
\text { the care provided to the } \\
\text { control group } \\
\text { - Performance bias: study did } \\
\text { not prevent patients from } \\
\text { seeing additional health care } \\
\text { providers } \\
\text { - Small sample size ( } n=23 \text { ) } \\
\text { - No objective measurement } \\
\text { of compliance with } \\
\text { antihypertensive medication } \\
\text { regimen } \\
\text { - Exclusively African-American } \\
\text { study population may affect } \\
\text { external validity }\end{array}$ \\
\hline Klein et al. $2009^{18}$ & $\begin{array}{l}\text { RCT, prospective } \\
\text { Inclusion from } \\
\text { September } 2003 \text { to } \\
\text { January } 2005\end{array}$ & $\begin{array}{l}\text { Germany: University } \\
\text { Hospital Mainz } \\
\text { Transplant surgery } \\
\text { unit }\end{array}$ & $\begin{array}{l}\text { Control }(C): n=24 \\
\text { Intervention }(\mathrm{I}) \text { : } \\
n=26 \\
\text { Mean age: } \\
\text { C: } 50.1 \text { years } \\
\text { I: } 52.8 \text { years } \\
\text { Sex, male: } \\
\text { C: } 54 \% \\
\text { I: } 54 \% \\
\text { Liver recipients }\end{array}$ & $\begin{array}{l}\text { Pharmaceutical care services: } \\
\text { - } 3 \text { or } 4 \text { meetings with } \\
\text { patients in the week before } \\
\text { discharge, for education } \\
\text { about immunosuppressive } \\
\text { therapy } \\
\text { - On discharge, provision of a } \\
\text { discharge medication plan, } \\
\text { written information about } \\
\text { the medication, and a diary } \\
\text { for laboratory data and } \\
\text { vital signs } \\
\text { - } 4 \text { to } 12 \text { meetings in the first } \\
\text { year after transplant to } \\
\text { discuss changes in } \\
\text { medication, laboratory } \\
\text { values, and other problems } \\
\text { - Drug therapy review }\end{array}$ & $\begin{array}{l}\text { - Contamination bias: patients } \\
\text { in the intervention and } \\
\text { control groups visited the } \\
\text { outpatient clinic at the same } \\
\text { time and were able to } \\
\text { exchange written and oral } \\
\text { information } \\
\text { - Performance bias: control } \\
\text { and intervention groups } \\
\text { received their immunosup- } \\
\text { pressant from the same } \\
\text { pharmacist, who had to } \\
\text { respond to questions and } \\
\text { problems from both groups } \\
\text { (for ethical reasons) } \\
\text { - Minimum threshold of } \\
\text { compliance rate to classify a } \\
\text { patient as "noncompliant" } \\
\text { was set arbitrarily, because it } \\
\text { is mostly unknown in } \\
\text { literature the point at which } \\
\text { noncompliance becomes } \\
\text { clinically relevant }\end{array}$ \\
\hline
\end{tabular}

the implementation of clinical pharmacy services in particular settings and locations. ${ }^{25}$

\section{Pharmacists' Activities}

The included studies reported a large range of pharmacist activities in solid organ transplant for both hospitalized and ambulatory patients, as described in Table 1 .

Alloway and others ${ }^{5}$ highlighted the following basic activities of the transplant pharmacist: dedicating time for the care of transplant recipient; attending daily rounds to evaluate pharmacotherapy; coordinating development and implementation of drug therapy protocols; providing medication 
Table 1 (part 2 of 4). Study Characteristics

\begin{tabular}{|c|c|c|c|c|c|}
\hline Reference & $\begin{array}{c}\text { Study Design } \\
\text { and Timeframe }\end{array}$ & Setting & $\begin{array}{c}\text { Sample Size } \\
\text { and Patient } \\
\text { Characteristics }\end{array}$ & $\begin{array}{l}\text { Pharmacists' } \\
\text { Interventions }\end{array}$ & Bias \\
\hline $\begin{array}{l}\text { Chisholm-Burns } \\
\text { et al. } 2013^{17}\end{array}$ & $\begin{array}{l}\text { RCT, prospective } \\
\text { January } 2010 \text { to } \\
\text { November } 2012\end{array}$ & $\begin{array}{l}\text { United States: } \\
\text { Avella Specialty } \\
\text { Pharmacy (specialty } \\
\text { pharmacy network), } \\
\text { multicentre }\end{array}$ & $\begin{array}{l}\text { Control }(C): n=74 \\
\text { Intervention }(\mathrm{I}): \\
n=76 \\
\text { Mean age } \pm \text { SD: } \\
\text { C: } 51.32 \pm 13.69 \\
\text { years } \\
\text { l: } 52.78 \pm 13.55 \text { years } \\
\text { Sex, male: } \\
\text { C: } 55.4 \% \\
\text { l: } 56.6 \% \\
\text { Kidney recipients }\end{array}$ & $\begin{array}{l}\text { Semistructured } 20 \text { - to } 30 \text {-min } \\
\text { meetings with patients at } \\
0,3,6,9 \text {, and } 12 \text { months } \\
\text { to sign or renew an } \\
\text { adherence-promoting } \\
\text { behavioural contract and } \\
\text { discuss its } 6 \text { components: } \\
\text { - Goal-setting } \\
\text { - Motivation } \\
\text { - Social support } \\
\text { - Memory techniques } \\
\text { - Problem-solving } \\
\text { - Consequences of } \\
\text { nonadherence }\end{array}$ & $\begin{array}{l}\text { - No "attention" control } \\
\text { group receiving interactions } \\
\text { with a study pharmacist } \\
\text { without the behavioural } \\
\text { contract } \\
\text { - A single pharmacist } \\
\text { performed the intervention, } \\
\text { limiting generalizability } \\
\text { - No direct collection of } \\
\text { utilization and cost data, } \\
\text { although the methods used } \\
\text { (self-report and Medicare } \\
\text { Expenditure Panel Survey) } \\
\text { have been validated } \\
\text { - No measurement of } \\
\text { self-efficacy } \\
\text { - White, Hispanic, and female } \\
\text { patients were over- } \\
\text { represented in the study } \\
\text { compared with the general } \\
\text { United States population }\end{array}$ \\
\hline \multicolumn{6}{|c|}{ Quasi-randomized controlled trial } \\
\hline Joost et al. $2014^{11}$ & $\begin{array}{l}\text { Quasi-randomized } \\
\text { controlled trial, } \\
\text { prospective } \\
\text { August } 2008 \text { to } \\
\text { July } 2010\end{array}$ & $\begin{array}{l}\text { Germany: Erlangen } \\
\text { University Hospital } \\
\text { Outpatient clinic of } \\
\text { Department of } \\
\text { Nephrology and } \\
\text { Hypertension }\end{array}$ & $\begin{array}{l}\text { Control }(C): n=39 \\
\text { Intervention }(\mathrm{I}): \\
n=35 \\
\text { Mean age } \pm \text { SD: } \\
\text { C: } 54 \pm 11.9 \text { years } \\
\text { I: } 51 \pm 13.3 \text { years } \\
\text { Sex, male: } \\
\text { C: } 62 \% \\
\text { l: } 77 \% \\
\text { Kidney recipients }\end{array}$ & $\begin{array}{l}\text { - } 3 \text { standardized counselling } \\
\text { sessions of } 30 \text { min each } \\
\text { within first } 2 \text { weeks after } \\
\text { transplant } \\
\text { - } 1 \text { to } 3 \text { quarterly follow-up } \\
\text { counselling sessions over } \\
12 \text { months } \\
\text { - Additional pharmaceutical } \\
\text { care over phone or by } \\
\text { email when necessary }\end{array}$ & $\begin{array}{l}\text { - Selection bias: only } 40 \% \text { of } \\
\text { eligible transplant patients } \\
\text { agreed to participate in the } \\
\text { study (nonadherence could } \\
\text { be a cause of refusal) } \\
\text { - One-year time horizon: } \\
\text { the results cannot be } \\
\text { extrapolated beyond } 1 \text { year } \\
\text { - Contamination bias: patients } \\
\text { in the intervention group } \\
\text { may have shared their } \\
\text { new-found knowledge with } \\
\text { patients in control group }\end{array}$ \\
\hline \multicolumn{6}{|l|}{ Cohort studies } \\
\hline $\begin{array}{l}\text { Harrison et al. } \\
2012^{14}\end{array}$ & $\begin{array}{l}\text { Cohort study, } \\
\text { prospective } \\
\text { Control: November } \\
2007 \text { to June } 2008 \\
\\
\text { Intervention: } \\
\text { July } 2008 \text { to } \\
\text { January } 2009\end{array}$ & $\begin{array}{l}\text { Canada: Toronto } \\
\text { General Hospital } \\
\text { Outpatient lung } \\
\text { transplant clinic }\end{array}$ & $\begin{array}{l}\text { Control (C): } n=43 \\
\text { Intervention (I): } \\
n=43 \\
\text { Age (years): } \\
\text { 18-39: } 30 \% \\
\text { (C) vs } 12 \%(\text { (I) } \\
\text { 40-59: } 47 \% \\
\text { (C) vs } 51 \%(I) \\
\geq 60: 23 \% \text { (C) vs } \\
37 \% \text { (I) } \\
\text { Sex, male: } \\
\text { C: } 56 \% \\
\text { l: } 56 \% \\
\text { Lung recipients }\end{array}$ & $\begin{array}{l}\text { - Primary pharmaceutical } \\
\text { care intervention (drug } \\
\text { therapy review, therapeutic } \\
\text { recommendations) } \\
\text { - Patient teaching } \\
\text { - Medication reconciliation } \\
\text { - Referral of issue for } \\
\text { team follow-up } \\
\text { - Optimization of medication } \\
\text { adherence } \\
\text { - Medication information } \\
\text { and advice for patients and } \\
\text { the team } \\
\text { - Assistance with drug } \\
\text { coverage issues } \\
\text { - Collaboration with } \\
\text { community pharmacists }\end{array}$ & $\begin{array}{l}\text { - Performance bias: clinicians } \\
\text { may have not performed a } \\
\text { comprehensive drug therapy } \\
\text { assessment, knowing that } \\
\text { patients would be subject } \\
\text { to subsequent pharmacist } \\
\text { reviews } \\
\text { - Inconsistencies of } \\
\text { intervention: pharmacists in } \\
\text { the study received no } \\
\text { formalized training in } \\
\text { outpatient practice } \\
\text { - Most patients met with } \\
\text { pharmacist only once during } \\
\text { the timeframe of the study } \\
\text { (additional visits over a } \\
\text { longer period might lead to } \\
\text { greater impact on patient } \\
\text { care outcomes) }\end{array}$ \\
\hline
\end{tabular}

continued on page 329

reconciliation, medication therapy management, and discharge counselling; providing education to members of the transplant team; facilitating cost and pharmacotherapy optimization to maximize patient outcomes; providing transplant medication education to patients; leading and assisting with clinical and pharmacoeconomic research; and providing 24/7 pharmaco- therapeutic support. This list strongly concurs with the interventions summarized in Table 1 of this review, except for research. Indeed, the most frequently reported activities in studies included in our review were patient education and counselling, reviewing and optimizing drug therapy, and medication reconciliation or medical history. 
Table 1 (part 3 of 4). Study Characteristics

\begin{tabular}{|c|c|c|c|c|c|}
\hline Reference & $\begin{array}{l}\text { Study Design } \\
\text { and Timeframe }\end{array}$ & Setting & $\begin{array}{c}\text { Sample Size } \\
\text { and Patient } \\
\text { Characteristics }\end{array}$ & $\begin{array}{l}\text { Pharmacists' } \\
\text { Interventions }\end{array}$ & Bias \\
\hline $\begin{array}{l}\text { Maldonado et al. } \\
2013^{12}\end{array}$ & $\begin{array}{l}\text { Cohort study, } \\
\text { retrospective } \\
\text { Control cohort: } 2007 \\
\text { Intervention cohort: } \\
2011\end{array}$ & $\begin{array}{l}\text { United States: } \\
\text { Providence Sacred } \\
\text { Heart Medical } \\
\text { Center \& Children's } \\
\text { Hospital } \\
\text { Inpatient and } \\
\text { outpatient } \\
\text { transplantation } \\
\text { clinic }\end{array}$ & $\begin{array}{l}\text { Control }(C): n=60 \\
\text { Intervention }(I): \\
n=54 \\
\text { Mean age at } \\
\text { transplant: } \\
\text { C: } 51.4 \text { years } \\
\text { I: } 55.0 \text { years } \\
\text { Sex, male: } \\
\text { C: } 65 \% \\
\text { I: } 63 \% \\
\text { Kidney recipients }\end{array}$ & $\begin{array}{l}\text { - Daily rounds with the } \\
\text { interdisciplinary team } \\
\text { - Pharmacotherapy } \\
\text { recommendations to } \\
\text { physicians, surgeons, and } \\
\text { midlevel practitioners } \\
\text { - Active drug monitoring } \\
\text { - Medication reconciliation } \\
\text { and discharge planning } \\
\text { - Patient education }\end{array}$ & $\begin{array}{l}\text { - Performance bias: changes } \\
\text { in usage of anti-thymocyte } \\
\text { globulin induction therapy, } \\
\text { a new program director, } \\
\text { and addition of a transplant } \\
\text { nurse practitioner may have } \\
\text { influenced the results } \\
\text { - No assessment of patient } \\
\text { health literacy or medication } \\
\text { compliance, which are } \\
\text { viewed as the primary } \\
\text { contribution of transplant } \\
\text { pharmacists }\end{array}$ \\
\hline $\begin{array}{l}\text { Musgrave et al. } \\
2013^{13}\end{array}$ & $\begin{array}{l}\text { Cohort study, } \\
\text { prospective } \\
\text { Retrospective cohort: } \\
2006 \text { to } 2008 \\
\text { Prospective cohort: } \\
2011\end{array}$ & $\begin{array}{l}\text { United States: } \\
\text { Medical University of } \\
\text { South Carolina } \\
\text { Department of } \\
\text { Transplant Surgery }\end{array}$ & $\begin{array}{l}\text { Retrospective cohort } \\
\text { (C): } n=128 \\
\text { Prospective cohort } \\
\text { (I): } n=64 \\
\text { Median age: } \\
\text { C: } 51.5 \text { years } \\
\text { I: } 54 \text { years } \\
\text { Sex, male: } \\
\text { C: } 65.6 \% \\
\text { I: } 68.8 \% \\
\text { Abdominal } \\
\text { transplant patients }\end{array}$ & $\begin{array}{l}\text { - At discharge, 5-30 min } \\
\text { (median } 15 \text { min) spent per } \\
\text { patient to verify medication } \\
\text { reconciliation } \\
\text { - At the first follow-up } \\
\text { appointment (next business } \\
\text { day following discharge), } \\
\text { 0-90 min (median } 20 \text { min) } \\
\text { spent per patient to review } \\
\text { medications } \\
\text { - Prevention and/or correction } \\
\text { of the identified drug-related } \\
\text { problems }\end{array}$ & $\begin{array}{l}\text { - Chart review to identify } \\
\text { errors was done with } \\
\text { retrospective records, which } \\
\text { do not always provide } \\
\text { explanations for changes } \\
\text { that might seem like errors } \\
\text { but could have been } \\
\text { intentional } \\
\text { - Analysis bias: chart review } \\
\text { was conducted by a single } \\
\text { reviewer } \\
\text { - Analysis bias: classification } \\
\text { of errors by severity was } \\
\text { performed by a single } \\
\text { reviewer (but this was } \\
\text { controlled by use of a } \\
\text { validated rating tool) } \\
\text { - In the retrospective period, } \\
\text { no correlation of the errors } \\
\text { to detrimental clinical } \\
\text { outcomes }\end{array}$ \\
\hline Tschida et al. $2013^{20}$ & $\begin{array}{l}\text { Cohort study, } \\
\text { retrospective } \\
\text { Inclusion from } \\
\text { August } 2007 \text { to } \\
\text { December } 2007\end{array}$ & $\begin{array}{l}\text { United States: } \\
\text { United Healthcare } \\
\text { Pharmacy (specialty } \\
\text { pharmacy network), } \\
\text { multicentre }\end{array}$ & $\begin{array}{l}\text { Retail pharmacy } \\
\text { group }(C): n=519 \\
\text { Specialty pharmacy } \\
\text { group }(I): n=519 \\
\text { Mean age (years): } \\
\text { C: } 49.78 \text { years } \\
\text { I: } 49.78 \text { years } \\
\text { Sex, male: } \\
\text { C: } 62 \% \\
\text { I: } 61 \% \\
\text { Renal transplant } \\
\text { patients }\end{array}$ & $\begin{array}{l}\text { Transplant medication } \\
\text { specialty pharmacy program: } \\
\text { - Monthly face-to-face } \\
\text { consultations for the first } 3 \\
\text { months after transplant, } \\
\text { then about every } 3 \text { months } \\
\text { - Additional clinical } \\
\text { counselling sessions by phone } \\
\text { - Provision of clinical expertise } \\
\text { and patient education in } \\
\text { transplant medications and } \\
\text { comorbid conditions } \\
\text { - Monthly refill reminders, } \\
\text { adherence screening } \\
\text { (intervention with physician } \\
\text { if necessary) } \\
\text { - 24/7 pharmacist support } \\
\text { available to patient }\end{array}$ & $\begin{array}{l}\text { - Selection bias: patients may } \\
\text { have self-selected into either } \\
\text { the specialty or retail } \\
\text { pharmacy benefit programs } \\
\text { (sicker patients may have } \\
\text { differentially chosen one } \\
\text { type of pharmacy over the } \\
\text { other) } \\
\text { - Adherence estimations using } \\
\text { retrospective data do not } \\
\text { always give an accurate } \\
\text { representation of whether } \\
\text { the medication was taken } \\
\text { exactly as prescribed } \\
\text { - No measurement of how } \\
\text { consistently and how many } \\
\text { patients participated in the } \\
\text { pharmacy consultations on } \\
\text { an ongoing basis of monthly } \\
\text { and every } 3 \text { months } \\
\text { meetings }\end{array}$ \\
\hline
\end{tabular}

continued on page 330

Patient education and teaching sessions aimed to educate patients about all aspects of their medications and the risks of nonadherence, and to answer questions. Handing out information sheets and providing support by phone or e-mail were activities performed by pharmacists in many of the studies.
Nonadherence to the immunosuppressive regimen after transplant is a major issue than can lead to serious outcomes, such as transplant rejection or even death. Clinical pharmacists can improve patient adherence to medications. ${ }^{15}$ In a unique approach, Chisholm-Burns and others ${ }^{17}$ used a behavioural 
Table 1 (part 4 of 4). Study Characteristics

\begin{tabular}{|c|c|c|c|c|c|}
\hline Reference & $\begin{array}{l}\text { Study Design } \\
\text { and Timeframe }\end{array}$ & Setting & $\begin{array}{c}\text { Sample Size } \\
\text { and Patient } \\
\text { Characteristics }\end{array}$ & $\begin{array}{l}\text { Pharmacists' } \\
\text { Interventions }\end{array}$ & Bias \\
\hline \multicolumn{6}{|l|}{ Pre-post studies } \\
\hline Partovi et al. $1995^{22}$ & $\begin{array}{l}\text { Pre-post study, } \\
\text { prospective } \\
\text { March to June } 1993\end{array}$ & $\begin{array}{l}\text { Canada: Vancouver } \\
\text { Hospital and Health } \\
\text { Sciences Centre } \\
\text { Solid organ } \\
\text { transplant clinic }\end{array}$ & $\begin{array}{l}\text { Group: } n=28 \\
\text { Mean age: } \\
47.2 \text { years } \\
\text { Sex, male: } 43 \% \\
\text { Solid organ } \\
\text { recipients }\end{array}$ & $\begin{array}{l}\text { Medication counselling } \\
\text { program: } \\
\text { - Oral counselling by a } \\
\text { pharmacist and provision of } \\
\text { medication teaching sheets } \\
\text { (step 1) } \\
\text { - Patient participation in } \\
\text { self-medication program } \\
\text { (step 2) } \\
\text { Four identical tests given to } \\
\text { patients throughout the } \\
\text { program to evaluate } \\
\text { knowledge retention: } \\
\text { - Pre-test (just before step 1) } \\
\text { - Post-test } 1 \text { (2-3 days } \\
\text { after step 1) } \\
\text { - Post-test 2 (3-5 days } \\
\text { after step 2) } \\
\text { - Post-test } 3 \text { (5-7 days } \\
\text { after post-test 2) }\end{array}$ & $\begin{array}{l}\text { - Only short-term knowledge } \\
\text { retention was assessed } \\
\text { - Inconsistency of the quality } \\
\text { of teaching provided by } \\
\text { each of the } 4 \text { pharmacists } \\
\text { involved in the counselling } \\
\text { and testing } \\
\text { - Confounding factors: } \\
\text { patients who had health- } \\
\text { related jobs scored higher; } \\
\text { central nervous system } \\
\text { depressive drugs lowered } \\
\text { test performance } \\
\text { - No control group }\end{array}$ \\
\hline $\begin{array}{l}\text { Chisholm et al. } \\
2007^{19}\end{array}$ & $\begin{array}{l}\text { Pre-post study, } \\
\text { retrospective } \\
\text { Inclusion from } \\
\text { November } 1999 \text { to } \\
\text { September } 2005\end{array}$ & $\begin{array}{l}\text { United States: } \\
\text { Medical College of } \\
\text { Georgia } \\
\text { Renal transplant } \\
\text { clinic }\end{array}$ & $\begin{array}{l}\text { Group: } n=36 \\
\text { Mean age } \pm \text { SD: } \\
52.78 \pm 13.37 \text { years } \\
\text { Sex, male: } 61.1 \% \\
\text { Kidney recipients }\end{array}$ & $\begin{array}{l}\text { Medication therapy } \\
\text { management services } \\
\text { (provided at least once } \\
\text { a month): } \\
\text { - Review of medication } \\
\text { profile to ensure therapeutic } \\
\text { outcomes and minimize } \\
\text { adverse drug events } \\
\text { - Identify, resolve, and prevent } \\
\text { medication-related problems } \\
\text { - Interview patients } \\
\text { - Answer drug information } \\
\text { questions } \\
\text { - Make therapeutic } \\
\text { recommendations }\end{array}$ & $\begin{array}{l}\text { - No control group } \\
\text { - Small sample size } \\
(n=36)\end{array}$ \\
\hline Pinelli et al. $2014^{16}$ & $\begin{array}{l}\text { Pre-post study, } \\
\text { prospective } \\
2014\end{array}$ & $\begin{array}{l}\text { United States: } \\
\text { Henry Ford Hospital } \\
\text { Transplant institute }\end{array}$ & $\begin{array}{l}\text { Group: } n=22 \\
\text { Mean age } \pm \text { SD: } \\
59.3 \pm 9.5 \text { years } \\
\text { Sex, male: } 79 \% \\
\text { Kidney recipients }\end{array}$ & $\begin{array}{l}\text { Establishment of a pharmacist- } \\
\text { managed diabetes and } \\
\text { cardiovascular risk reduction } \\
\text { clinic (PMDC): } \\
\text { - 60-min appointment within } \\
7 \text { days of discharge by } \\
\text { inpatient transplant team } \\
\text { - 30-min follow-up } \\
\text { appointments at least } \\
\text { monthly over } 3 \text { months } \\
\text { - Disease state management } \\
\text { for diabetes, hypertension, } \\
\text { and dyslipidemia } \\
\text { - Standardized diabetes } \\
\text { self-management education } \\
\text { curriculum } \\
\text { - Referral to transplant } \\
\text { nutrition support services } \\
\text { as needed } \\
\text { - Medication reconciliation } \\
\text { at each visit } \\
\text { - Standardized discharge } \\
\text { process from PMDC at } 3 \\
\text { months to endocrinologist } \\
\text { or primary care provider }\end{array}$ & $\begin{array}{l}\text { - Small sample size }(n=22) \\
\text { - No control group }\end{array}$ \\
\hline
\end{tabular}

$\overline{\mathrm{RCT}}=$ randomized controlled trial, $\mathrm{SD}=$ standard deviation . 
Table 2 (part 1 of 3). Outcomes of Individual Studies

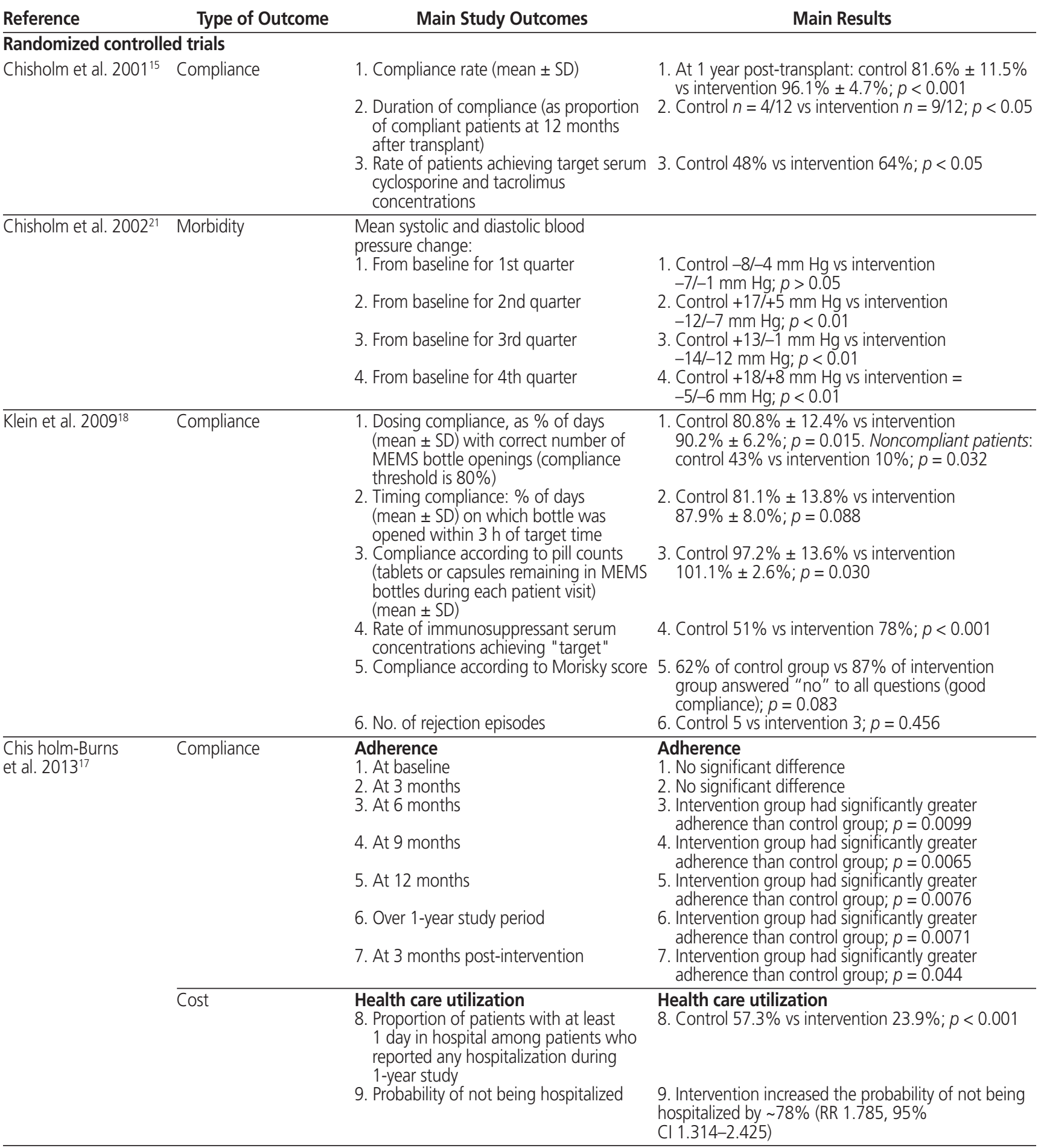

continued on page 332

contract and trimestral meetings to maximize patient adherence.

Reviewing and optimizing drug therapy helps in identifying, resolving and preventing drug-related problems. Musgrave and others ${ }^{13}$ reported a "significant" decrease of medication errors per patient at discharge because of pharmacist interventions. Chisholm and others ${ }^{15,19,21}$ also reported that pharmacist recommendations helped nephrologists to optimize prescriptions for transplant recipients.

Few of the included studies reported medication reconciliation. Nevertheless, this has been shown to be an essential component in optimizing the quality of prescriptions, prevent- 
Table 2 (part 2 of 3). Outcomes of Individual Studies

Reference

Quasi-randomized controlled trial
Joost et al. 2014

1. Daily adherence (as \% of days with correct dosing of MMF/MPA) during 1 -year monitoring period

2. Taking adherence (as \% of doses taken [bottle opening] compared with overall doses prescribed)

3. Timing adherence (as \% of doses taken within a 6 - $h$ interval $[ \pm 3 \mathrm{~h}]$ of standard intake time)

4. Adherence rate (as measured by pill count)

5. No. of drug holidays (defined as no MMF/MPA intake for $>48 \mathrm{~h}$ )

6. Adherence, as measured with Morisky questionnaire

7. Self-reported adherence
1. Control $57 \%(20 / 35)$ vs intervention $84 \%$ $(27 / 32) ; p=0.015$

2. Control $57 \%$ (20/35) vs intervention $84 \%$ $(27 / 32) ; p=0.015$

3. Control $86 \%(30 / 35)$ vs intervention $97 \%$ $(31 / 32) ; p=0.110$

4. Control $63 \%(22 / 35)$ vs intervention $84 \%$ $(27 / 32) ; p=0.047$

5. Control $43 \%(15 / 35)$ vs intervention $81 \%$ $(26 / 32) ; p=0.001$

6. Control $63 \%(22 / 35)$ vs intervention $63 \%$ $(20 / 32): p=0.695$

7. Control $77 \%(27 / 35)$ vs intervention $72 \%$ $(23 / 32) ; p=0.193$

\section{Cohort studies}

Harrison et al. 2012 $\quad$ Medication errors
1. No. of DTPs identified per visit (control 1. DTPs identified per: group, clinic visits; intervention group, - Intervention pharmacist visit: $1.05 \pm 1.34$ clinic visits and pharmacist visits) $($ mean \pm SD) relative to intervention pharmacist visit
- Intervention clinic visit $0.51 \pm 0.64 ; p=0.018$

- Control clinic visit $0.74 \pm 0.81 ; p=0.19$ relative to intervention pharmacist visit

\begin{tabular}{|c|c|c|c|}
\hline \multicolumn{2}{|c|}{ Maldonado et al. $2013^{12}$ Morbidity } & $\begin{array}{l}\text { 1. Mean hospital length of stay } \\
\text { 2. All cause } 30-, 90-\text {, and }>90-\text { day } \\
\text { readmission rates }\end{array}$ & $\begin{array}{l}\text { 1. Control (2007) } 7.8 \text { days vs } \\
\text { (2011) 3.4 days; } p<0.001 \\
\text { 2. No significant differences; } p \\
\text { comparisons }\end{array}$ \\
\hline \multirow{4}{*}{ Musgrave et al. $2013^{13}$} & \multirow{4}{*}{ Medication errors } & & \\
\hline & & $\begin{array}{l}\text { 1. No. of medication errors per patient } \\
\text { at discharge avoided through } \\
\text { pharmacist intervention (mean }+ \text { SD) }\end{array}$ & $\begin{array}{l}\text { 1. Retrospective } 0 \text { vs prospecti } \\
p<0.0001\end{array}$ \\
\hline & & $\begin{array}{l}\text { 2. No. of medication errors per patient } \\
\text { at discharge persisting until first } \\
\text { follow-up appointment (mean } \pm \text { SD) }\end{array}$ & $\begin{array}{l}\text { 2. Retrospective } 3.4 \pm 1.9 \text { vs } p \\
1.1 \pm 1.4 ; p<0.0001\end{array}$ \\
\hline & & $\begin{array}{l}\text { 3. \% of discharges with no } \\
\text { medication errors }\end{array}$ & $\begin{array}{l}\text { 3. Retrospective } 3.9 \% \text { vs pros } \\
p<0.0001\end{array}$ \\
\hline \multirow[t]{5}{*}{ Tschida et al. $2013^{20}$} & Cost & $\begin{array}{l}\text { 1. Mean total cost per patient in the } \\
\text { first follow-up year }\end{array}$ & $\begin{array}{l}\text { 1. } 13 \% \text { lower in the specialty } \\
(\$ 24315 \text { vs } \$ 27891) ; p=\end{array}$ \\
\hline & \multirow[t]{4}{*}{ Compliance } & $\begin{array}{l}\text { 2. Mean no. of oral transplant } \\
\text { prescriptions dispensed per patient } \\
\text { 3. Weighted medication possession ratio }\end{array}$ & $\begin{array}{l}\text { 2. Retail pharmacy group } 17.9 \\
\text { pharmacy group 18.67; } p< \\
\text { 3. Retail pharmacy group } 0.83 \\
\text { group 0.87: } p<0.0001\end{array}$ \\
\hline & & $\begin{array}{l}\text { 4. No. of patients with medication gap } \\
\text { (at least } 60 \text { days without } \\
\text { immunosuppressive drugs but followed } \\
\text { by re-initiation within study period) }\end{array}$ & $\begin{array}{l}\text { 4. Retail pharmacy group } 53 \mathrm{v} \\
\text { group } 29 ; p=0.006\end{array}$ \\
\hline & & $\begin{array}{l}\text { 5. No. of patients with discontinuation } \\
\text { (at least } 60 \text { days without } \\
\text { immunosuppressive drugs, never } \\
\text { followed by re-initiation within the } \\
\text { study period) }\end{array}$ & $\begin{array}{l}\text { 5. Retail pharmacy group } 104 \\
\text { pharmacy group 39; } p<0.6\end{array}$ \\
\hline & & $\begin{array}{l}\text { 6. Mean no. of dialysis-related inpatient } \\
\text { hospital stays per patient }\end{array}$ & $\begin{array}{l}\text { 6. Retail pharmacy group } 0.04 \\
\text { group } 0.02 ; p<0.03\end{array}$ \\
\hline \multicolumn{4}{|l|}{ Pre-post studies } \\
\hline Partovi et al. $1995^{22}$ & Other & $\begin{array}{l}\% \text { change in knowledge score (mean } \pm \text { SD } \\
\text { 1. Pre-test to post-test } 1 \\
\text { 2. Pre-test to post-test } 2 \\
\text { 3. Pre-test to post-test } 3 \\
\text { 4. Post-test } 1 \text { to post-test } 2 \\
\text { 5. Post-test } 2 \text { to post-test } 3\end{array}$ & $\begin{array}{l}\text { 1. } 24.8 \% \pm 10.6 \% ; p<0.05 \\
\text { 2. } 36.7 \% \pm 11.8 \% ; p<0.05 \\
\text { 3. } 40.9 \% \pm 12.7 \% ; p<0.05 \\
\text { 4. } 11.9 \% \pm 9.7 \% ; p<0.05 \\
\text { 5. } 4.21 \% \pm 8.9 \% ; p<0.05\end{array}$ \\
\hline
\end{tabular}


Table 2 (part 3 of 3). Outcomes of Individual Studies

Reference Type of Outcome

Main Study Outcomes

Main Results

\begin{tabular}{|c|c|c|c|}
\hline Chisholm et al. $2007^{19}$ & Morbidity & $\begin{array}{l}\text { 1. Clinical indicators for diabetes mellitus } \\
\text { (fasting blood glucose and HbA1c) } \\
\text { (mean } \pm \text { SD) } \\
\text { 2. Clinical indicators for hyperlipidemia } \\
\text { (LDL and total cholesterol) (mean } \pm \text { SD) } \\
\\
\text { 3. Clinical indicators for hypertension } \\
\text { (systolic and diastolic blood pressure) } \\
\text { (mean } \pm \text { SD) } \\
\text { 4. Serum tacrolimus concentration } \\
\text { (mean } \pm \text { SD) } \\
\\
\text { 5. Serum cyclosporine concentration } \\
\text { (mean } \pm \text { SD) }\end{array}$ & $\begin{array}{l}\text { 1. Fasting blood glucose: } 129.22 \pm 18.25 \mathrm{mg} / \mathrm{dL} \\
\text { (pre) vs } 112.22 \pm 17.43 \mathrm{mg} / \mathrm{dL} \text { (post); } p=0.001 \\
\text { HbA1c: } 8.07 \% \pm 0.81 \% \text { (pre) vs } 7.42 \% \pm 0.61 \% \\
\text { (post); } p=0.002 \\
\text { 2. LDL: } 305.48 \pm 66.20 \mathrm{mg} / \mathrm{dL} \text { (pre) vs } \\
\text { 191.78 } \pm 27.39 \mathrm{mg} / \mathrm{dL} \text { (post); } p<0.001 \\
\text { Total cholesterol: } 345.83 \pm 108.33 \mathrm{mg} / \mathrm{dL} \\
\text { (pre) vs } 239.91 \pm 47.24 \mathrm{mg} / \mathrm{dL} \text { (post); } p<0.001 \\
\text { 3. Systolic: } 140.52 \pm 7.81 \mathrm{~mm} \mathrm{Hg} \text { (pre) vs } \\
\text { 134.30 } \pm 7.54 \mathrm{~mm} \mathrm{Hg} \text { (post); } p<0.001 \\
\text { Diastolic: } 79.19 \pm 3.97 \mathrm{~mm} \mathrm{Hg} \text { (pre) vs } \\
77.04 \pm 4.24 \mathrm{~mm} \text { Hg (post); } p<0.001 \\
\text { 4. } 8.67 \pm 3.5 \mathrm{ng} / \mathrm{mL} \text { (pre) vs } 10.17 \pm 1.17 \mathrm{ng} / \mathrm{mL} \\
\text { (post); } p=0.343 \\
\text { No significant difference in no. of patients } \\
\text { achieving target concentrations } \\
\text { 5. } 178.77 \pm 61.4 \mathrm{ng} / \mathrm{mL} \text { (pre) vs } 214.7 \pm 44.14 \mathrm{ng} / \mathrm{mL} \\
\text { (post), } p=0.007 \\
\text { Significant improvement in no. of patients achieving } \\
\text { target concentrations; } p=0.008 \\
\text { 6. 0.50 } \pm 0.51 \text { (pre) vs } 0.22 \pm 0.42 \text { (post); } p=0.008 \\
\text { 7. Significantly increased scores for General Health, } \\
\text { Social Functioning, Role Emotional, Mental Health, } \\
\text { Physical Component Summary, and Mental } \\
\text { Component Summary scales; } p<0.01\end{array}$ \\
\hline \multirow[t]{3}{*}{ Pinelli et al. $2014^{16}$} & \multirow[t]{3}{*}{ Morbidity } & $\begin{array}{l}\text { HbA1c (mean } \pm \text { SD) } \\
\text { Intention-to-treat analysis } \\
\text { 1. At } 3 \text { months in patients with baseline } \\
\text { HbA1c }<7.0 \% \\
\text { 2. At } 6 \text { months in patients with baseline } \\
\text { HbA1c }<7.0 \% \\
\text { 3. At } 3 \text { months in patients with baseline } \\
\text { HbA1c } \geq 7.0 \% \\
\text { 4. At } 6 \text { months in patients with baseline } \\
\text { HbA1c } \geq 7.0 \%\end{array}$ & $\begin{array}{l}\text { HbA1c (mean } \pm \text { SD) } \\
\text { Intention-to-treat } \\
\text { 1. Baseline } 6.0 \% \pm 0.5 \% \text { vs } 3 \text { months } 6.6 \% \pm 0.9 \% \text {; } \\
p=0.20 \\
\text { 2. Baseline } 6.0 \% \pm 0.5 \% \text { vs } 6 \text { months } 6.2 \% \pm 0.6 \% \text {; } \\
p=0.48 \\
\text { 3. Baseline } 8.1 \% \pm 1.0 \% \text { vs } 3 \text { months } 7.3 \% \pm 1.2 \% \text {; } \\
p=0.07 \\
\text { 4. Baseline } 8.1 \% \pm 1.0 \% \text { vs } 6 \text { months } 7.5 \% \pm 0.8 \% \text {; } \\
p=0.16\end{array}$ \\
\hline & & Per protocol analysis & Per protocol analysis \\
\hline & & $\begin{array}{l}\text { 1. At } 3 \text { months in patients with baseline } \\
\text { HbA1c }<7.0 \% \\
\text { 2. At } 6 \text { months in patients with baseline } \\
\text { HbA1c }<7.0 \% \\
\text { 3. At } 3 \text { months in patients with baseline } \\
\text { HbA1c } \geq 7.0 \% \\
\text { 4. At } 6 \text { months in patients with baseline } \\
\text { HbA1c } \geq 7.0 \%\end{array}$ & $\begin{array}{l}\text { 1. Baseline } 6.0 \% \pm 0.5 \% \text { vs } 3 \text { months } 6.3 \% \pm 0.8 \% \text {; } \\
p=0.55 \\
\text { 2. Baseline } 6.0 \% \pm 0.5 \% \text { vs } 6 \text { months } 6.1 \% \pm 0.6 \% \text {; } \\
p=0.48 \\
\text { 3. Baseline } 8.3 \% \pm 1.0 \% \text { vs } 3 \text { months } 6.8 \% \pm 1.2 \% \text {; } \\
p=0.0041 \\
\text { 4. Baseline } 8.3 \% \pm 1.0 \% \text { vs } 6 \text { months } 7.5 \% \pm 1.0 \% \text {; } \\
p=0.15\end{array}$ \\
\hline
\end{tabular}

$\mathrm{Cl}=$ confidence interval, $\mathrm{DTP}=$ drug therapy problem, $\mathrm{HbA1C}=$ glycated hemoglobin, $\mathrm{LDL}=$ low-density lipoprotein,

MEMS = medication event monitoring system, MMF = mycophenolate mofetil, MPA = mycophenolic acid, RR = rate ratio, SD = standard deviation.

ing drug-related problems, and significantly reducing readmission rates in the emergency department. ${ }^{26}$ In the study by Maldonado and others, ${ }^{12}$ pharmacists proposed a discharge plan to the patients, in addition to performing medication reconciliation. Harrison and others ${ }^{14}$ described collaboration with community pharmacists; such collaborations reflect the importance of continuity of care between the transplant team and community practitioners to ensure an optimal prognosis. The development of telepharmacy tools may help with post-transplant home care.

\section{Outcomes}

Improvements in medication adherence, morbidity, costs, and medication errors were reported in the selected studies, but these outcomes were not linked to specific pharmacist activities.
There were clear benefits in terms of patient adherence to immunosuppressive treatments. ${ }^{11,15,17-20}$ Chisholm and others ${ }^{19}$ reported a significant reduction in transplant rejections from 1 year pre-enrollment to 1 year post-enrollment $(p=0.008)$. Klein and others ${ }^{18}$ found fewer rejection episodes in the intervention group, although the difference was not significant (small sample size). Three studies showed an increase in achievement of target serum concentrations of oral immunosuppressants. ${ }^{15,18,19}$

Significant positive outcomes were found in terms of comorbidities such as diabetes mellitus, hyperlipidemia, and hypertension, but the results were inconsistent for morbidity outcomes. ${ }^{16,19,21}$

In the study by Tschida and others, ${ }^{20}$ implementation of a transplant pharmacy program resulted in a significantly lower 
Table 3 (part 1 of 2). Rating of Pharmaceutical Interventions with DEPICT Tool ${ }^{10}$

Study (by Reference Number)

Element of Tool

\begin{tabular}{llllllllllll}
\hline 11 & 12 & 13 & 14 & 15 & 16 & 17 & 18 & 19 & 20 & 21 & 22 \\
\hline
\end{tabular}

A. Contact with the patient

1A. Face-to-face contact

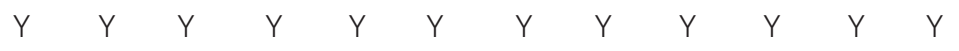

2A. Remote contact

$\begin{array}{lllllllllllll} & Y & N & N & N & Y & N & Y & N & N & Y & Y & N\end{array}$

\section{B. Timing of the intervention}

3B. At patient admission to a hospital, nursing home, or emergency department

4B. During hospital or nursing home stay

5B. At patient discharge or interfacility transfer

$6 \mathrm{~B}$. When a new or changed prescription is provided

7B. At the time of drug dispensing

$\begin{array}{llllllllllll}N & Y & N & N & N & N & N & N & N & N & N & N \\ Y & Y & N & N & N & N & N & Y & N & N & N & N \\ N & Y & Y & N & N & N & N & Y & N & N & N & N \\ N & N & N & N & N & N & N & N & N & N & N & N \\ N & N & N & N & N & N & N & N & N & N & N & N\end{array}$

\section{Setting of the intervention}

8C. Participant's home

9C. Community pharmacy

10C. Ambulatory or primary care setting co-located with medical services

11C. Independent ambulatory or primary care setting

12C. Hospital

13C. Long-term care facility

\begin{tabular}{llllllllllll}
$N$ & $N$ & $N$ & $N$ & $N$ & $N$ & $N$ & $N$ & $N$ & $Y$ & $N$ & $N$ \\
$N$ & $N$ & $N$ & $N$ & $N$ & $N$ & $Y$ & $N$ & $N$ & $Y$ & $N$ & $N$ \\
$Y$ & $Y$ & $N$ & $N$ & $N$ & $Y$ & $N$ & $N$ & $N$ & $N$ & $N$ & $N$ \\
$N$ & $N$ & $N$ & $N$ & $N$ & $N$ & $N$ & $N$ & $N$ & $N$ & $N$ & $N$ \\
$Y$ & $Y$ & $Y$ & $Y$ & $Y$ & $N$ & $N$ & $Y$ & $N$ & $N$ & $Y$ & $Y$ \\
$N$ & $N$ & $N$ & $N$ & $N$ & $N$ & $N$ & $N$ & $N$ & $N$ & $N$ & $N$ \\
\hline
\end{tabular}

\section{Target population}

14D. Condition-specific intervention

15D. Population-specific intervention

\begin{tabular}{llllllllllll}
$Y$ & $Y$ & $Y$ & $Y$ & $Y$ & $Y$ & $Y$ & $Y$ & $Y$ & $Y$ & $Y$ & $Y$ \\
$Y$ & $Y$ & $Y$ & $Y$ & $Y$ & $Y$ & $Y$ & $Y$ & $Y$ & $Y$ & $Y$ & $Y$ \\
\hline
\end{tabular}

\section{E. Clinical data sources}

16E. All current medications in use by the patient

17E. Pharmacy or dispensing records

18E. Laboratory tests or drug monitoring data

19E. Disease self-monitoring data

20E. Patient's physical or functional assessment

21E. Medical records

22E. Patient interview (anamnesis)

\section{F. What is assessed}

23F. Medication-use process (errors)

24F. Legal or administrative aspects of drug prescriptions

25F. Patient's knowledge, health literacy,

or communication skills

26F. Patient's adherence to treatment

27F. Health outcomes

28F. Patient's quality of life

29F. Patient's satisfaction

30F. Costs of treatment

\section{G. Pharmacist's autonomy to perform an action}

31G. Change dosage regimen

32G. Suspend medication

33G. Start a new medication

34G. Order laboratory tests or perform drug monitoring

\section{H. Pharmacist communication}

$35 \mathrm{H}$. Directly with the patient

$36 \mathrm{H}$. With the physician or health care team

$37 \mathrm{H}$. Written recommendations to the physician or health care team

38H. Face-to-face or telephone recommendations to the physician of health care team

$\begin{array}{llllllllllll}N & Y & Y & Y & Y & Y & N & Y & Y & Y & Y & N \\ N & N & Y & N & Y & N & Y & N & N & N & Y & N \\ N & Y & N & N & Y & N & N & N & N & N & Y & N \\ N & N & N & N & N & N & N & N & N & N & N & N \\ N & N & N & Y & N & N & N & N & N & N & N & N \\ N & Y & N & N & Y & N & N & N & N & N & Y & N \\ N & N & N & N & N & N & N & N & N & N & N & N\end{array}$

\begin{tabular}{llllllllllll}
$N$ & $N$ & $Y$ & $Y$ & $N$ & $N$ & $N$ & $N$ & $N$ & $N$ & $N$ & $N$ \\
$N$ & $N$ & $N$ & $N$ & $N$ & $N$ & $N$ & $N$ & $N$ & $N$ & $N$ & $N$ \\
$N$ & $N$ & $N$ & $N$ & $N$ & $N$ & $N$ & $N$ & $N$ & $N$ & $N$ & $Y$ \\
$Y$ & $N$ & $N$ & $N$ & $Y$ & $N$ & $Y$ & $Y$ & $Y$ & $Y$ & $N$ & $N$ \\
$N$ & $Y$ & $N$ & $N$ & $N$ & $Y$ & $N$ & $N$ & $Y$ & $N$ & $Y$ & $N$ \\
$N$ & $N$ & $N$ & $N$ & $N$ & $N$ & $N$ & $N$ & $Y$ & $N$ & $N$ & $N$ \\
$N$ & $N$ & $N$ & $Y$ & $N$ & $N$ & $N$ & $N$ & $N$ & $N$ & $N$ & $N$ \\
$N$ & $N$ & $N$ & $N$ & $N$ & $N$ & $N$ & $N$ & $Y$ & $Y$ & $N$ & $N$ \\
\hline
\end{tabular}

\begin{tabular}{|c|c|c|c|c|c|c|c|c|c|c|c|}
\hline $\mathrm{N}$ & $\mathrm{N}$ & $\mathrm{N}$ & $\mathrm{N}$ & $\mathrm{N}$ & $\mathrm{N}$ & $\mathrm{N}$ & $\mathrm{N}$ & $\mathrm{N}$ & $\mathrm{N}$ & $\mathrm{N}$ & $\mathrm{N}$ \\
\hline$N$ & $\mathrm{~N}$ & $\mathrm{~N}$ & $\mathrm{~N}$ & $\mathrm{~N}$ & $\mathrm{~N}$ & $\mathrm{~N}$ & $N$ & $N$ & $\mathrm{~N}$ & $\mathrm{~N}$ & $\mathrm{~N}$ \\
\hline $\mathrm{N}$ & $\mathrm{N}$ & $N$ & $\mathrm{~N}$ & $\mathrm{~N}$ & $\mathrm{~N}$ & $\mathrm{~N}$ & $\mathrm{~N}$ & $N$ & $\mathrm{~N}$ & $\mathrm{~N}$ & $\mathrm{~N}$ \\
\hline $\mathrm{N}$ & $Y$ & $N$ & $\mathrm{~N}$ & $\mathrm{~N}$ & $\mathrm{~N}$ & $\mathrm{~N}$ & $\mathrm{~N}$ & $\mathrm{~N}$ & $\mathrm{~N}$ & $\mathrm{~N}$ & $\mathrm{~N}$ \\
\hline
\end{tabular}

$\begin{array}{llllllllllll}Y & Y & N & Y & Y & Y & Y & Y & Y & Y & Y & Y \\ N & Y & N & Y & Y & Y & N & N & Y & N & Y & N \\ N & N & N & Y & N & N & N & N & N & N & N & N \\ N & N & N & Y & N & N & N & N & N & N & N & N\end{array}$


Table 3 (part 2 of 2). Rating of Pharmaceutical Interventions with DEPICT Tool ${ }^{10}$

Study (by Reference Number)

Element of Tool

\begin{tabular}{llllllllllll}
11 & 12 & 13 & 14 & 15 & 16 & 17 & 18 & 19 & 20 & 21 & 22 \\
\hline
\end{tabular}

I. Support resources provided by the pharmacist

391. A patient's medication list to the physician

40l. A medication list or summary to the patient

411. Written, video, or audio educational material to the patient

42I. Medication adherence or administration aid

43I. Disease self-management diary

J. Education and counselling

44J. Disease-specific or medication counselling to the patient

45J. Lifestyle or self-management education to the patient

46J. Education program to a group of patients

\begin{tabular}{llllllllllll}
$N$ & $Y$ & $N$ & $N$ & $N$ & $N$ & $N$ & $N$ & $N$ & $N$ & $N$ & $N$ \\
$N$ & $Y$ & $N$ & $Y$ & $N$ & $N$ & $N$ & $Y$ & $N$ & $N$ & $N$ & $N$ \\
$Y$ & $N$ & $N$ & $N$ & $Y$ & $N$ & $N$ & $N$ & $N$ & $N$ & $Y$ & $Y$ \\
$Y$ & $Y$ & $N$ & $Y$ & $N$ & $N$ & $N$ & $N$ & $N$ & $N$ & $N$ & $N$ \\
$N$ & $N$ & $N$ & $N$ & $N$ & $N$ & $N$ & $Y$ & $N$ & $N$ & $N$ & $N$ \\
\hline
\end{tabular}

\section{Follow-up}

47L. Focus on medication-use process

48L. Focus on health or therapeutic outcomes

49L. Follow-up is performed through face-to-face encounters

50L. Follow-up is performed through remote contacts

$51 \mathrm{~L}$. Duration of the follow-up (write the number of months)

\section{Other actions}

52M. Screening for disease risk factors

53M. Development of a drug formulary, guideline,

or clinical protocol

54M. Provider or prescriber education

DEPICT score*

$\begin{array}{llllllllllll}Y & Y & N & Y & Y & Y & N & Y & N & Y & Y & Y \\ N & N & N & N & N & N & Y & Y & N & N & N & N \\ N & N & N & N & N & N & N & N & N & N & N & N\end{array}$

$\mathrm{N}=$ no (item not reported in study), $\mathrm{Y}=$ yes (item reported in study).

*For each of the 12 sections, a score of 1 was assigned if the reviewers answered "yes" to at least one element of the section.

The number of sections with a score of 1 was summed to generate the overall DEPICT score (maximum 12).

mean total cost per patient ( $\$ 24315$ versus $\$ 27891,13 \%$ decrease; $p=0.03$ ), which the authors attributed mainly to a significantly lower mean transplant-related medical cost $(\$ 5960$ versus $\$ 8486,30 \%$ decrease; $p=0.04$ ).

Musgrave and others ${ }^{13}$ described the avoidance of discharge medication errors through pharmacist intervention, a decrease in discharge medication errors per patient persisting until the first follow-up appointment, and a greatly improved percentage of discharges with no medication errors. Harrison and others ${ }^{14}$ reported a decrease in the mean number of drug therapy problems identified per visit.

Patients' knowledge of medications was appraised in only one study. ${ }^{22}$ The benefits for short-term information retention were significant, but the study did not examine long-term retention. Given that patient motivation and care intensity often diminish with time, long-term persistence of pharmacistinduced outcomes needs to be evaluated.

\section{Description of Pharmaceutical Interventions}

In studies designed to evaluate the roles and impacts of health care professionals, it is very important to have a clear and complete description of the intervention. Associating an intervention with specific outcomes is especially difficult where multidisciplinary teams are involved. According to the DEPICT tool, ${ }^{10}$ the descriptions of the interventions in the included studies were generally of good quality. Nonetheless, more complete descriptions should be provided in future studies, especially regarding the timing of the intervention and pharmacists' autonomy.

As for most pharmacy practice research studies, the studies included in this review had small sample sizes, some had no control group $(n=3)$, and the interventions were insufficiently described to be fully reproducible. Usual sources of bias were reported, including performance bias and contamination bias. In clinical practice within a hospital, it is usually difficult to eliminate these 2 types of bias.

\section{Transplant Pharmacy Training}

Transplant recipients are treated with multiple drugs, including medications with a narrow therapeutic index. It was therefore surprising to find only a limited number of articles describing pharmacists' roles and outcomes in this area. This systematic review highlights the need to structure teaching and internships in this discipline and to further document the practice of pharmacists in transplant medicine. Professional specialty networks may certainly contribute to better training, 
organization, and documentation. For instance, the American Society for Transplantation has a transplant pharmacy community of practice. ${ }^{27}$ In addition, the American College of Clinical Pharmacy has an immunology/transplantation practice and research network. ${ }^{28}$ The Board of Pharmacy Specialties received a petition to recognize solid organ transplantation pharmacy as a new specialty; the Board's public comment period on this petition closed on May 15, 2018. ${ }^{29}$

In Canada, the Canadian Society of Transplantation has a pharmacist group whose mission is to "provide leadership and a collaborative forum for the advancement of pharmacist clinical practice in transplantation and pharmacist-led research and education" ${ }^{30}$ The Canadian Society of Hospital Pharmacists has a transplant Pharmacy Specialty Network that promotes "practice excellence and the enhancement of patient-centred pharmacy practice through information sharing, educational events, and the facilitation of research for pharmacists who are interested in the area of transplant pharmacy practice (solid organ and hematopoietic stem cell transplant)". ${ }^{31}$

There is currently no published literature about transplantspecific training offered in pharmacy, in Canada or elsewhere. Such training may vary substantially among regions and programs, which may explain the paucity of data as well as the wide variety of roles described in the literature.

\section{Limitations}

The systematic literature search was conducted in only 4 databases, and all articles published in a language other than English or French were excluded. As a result, some eligible studies may have gone undetected. Although descriptive results lack statistical proof of significance, they may carry compelling information that could prove useful in establishing a more accurate image of the roles and impacts of the pharmacist. However, for practical reasons (notably the difficulty of screening for quality), they were omitted from this review. Eight studies involved kidney transplant recipients exclusively, and the 4 remaining studies were spread among recipients of abdominal, liver, lung, and unspecified transplants. Most antirejection medications are lifelong treatments, yet the temporal horizon was limited to a year or less in virtually all of the studies. It is unknown whether pharmacist interventions have lasting effects, especially in the case of temporary activities. It would be interesting to explore which interventions were the most time-effective.

\section{CONCLUSION}

Currently available evidence suggests that pharmacists can improve patient outcomes in solid organ transplant settings. Adherence, morbidity, costs, and medication errors were identified as the main outcomes that were improved by pharmaceutical interventions. Transplant programs need to invest more in this resource.
References

1. Annual statistics on organ replacement in Canada: dialysis, transplantation and donation, 2007 to 2016. Ottawa (ON): Canadian Institute for Health Information; 2017 [cited 2018 May 17]. Available from: https:// www.cihi.ca/sites/default/files/document/corr_ar-snapshot-en.pdf

2. Denhaerynck K, Dobbels F, Cleemput I, Desmyttere A, Schäfer-Keller P, Schaub S, et al. Prevalence, consequences, and determinants of nonadherence in adult renal transplant patients: a literature review. Transpl Int. 2005; 18(10):1121-33.

3. Pinsky BW, Takemoto SK, Lentine KL, Burroughs TE, Schnitzler MA, Salvalaggio PR. Transplant outcomes and economic costs associated with patient noncompliance to immunosuppression. Am J Transplant. 2009; 9(11):2597-606.

4. Mitchell JF. Pharmacist involvement as a member of a renal transplant team. Am J Hosp Pharm. 1976;33(1):55-8.

5. Alloway RR, Dupuis R, Gabardi S, Kaiser TE, Taber DJ, Tichy EM, et al. Evolution of the role of the transplant pharmacist on the multidisciplinary transplant team. Am J Transplant. 2011;11(8):1576-83.

6. Singhal PK, Raisch DW, Gupchup GV. The impact of pharmaceutical services in community and ambulatory care settings: evidence and recommendations for future research. Ann Pharmacother. 1999;33(12):1336-55.

7. Melchiors AC, Correr CJ, Venson R, Pontarolo R. An analysis of quality of systematic reviews on pharmacist health interventions. Int J Clin Pharm. 2012;34(1):32-42.

8. Charrois TL, Durec T, Tsuyuki RT. Systematic reviews of pharmacy practice research: methodologic issues in searching, evaluating, interpreting, and disseminating results. Ann Pharmacother. 2009;43(1):118-22.

9. Moher D, Liberati A, Tetzlaff J, Altman DG. PRISMA 2009 checklist. PRISMA Group; 2009 [cited 2016 Sep 20]. Available from: http://prismastatement.org/documents/PRISMA\%202009\%20checklist.pdf

10. Correr CJ, Melchiors AC, de Souza TT, Rotta I, Salgado TM, FernandezLlimos F. A tool to characterize the components of pharmacist interventions in clinical pharmacy services: the DEPICT project. Ann Pharmacother. 2013;47(7-8):946-52.

11. Joost R, Dörje F, Schwitulla J, Eckardt KU, Hugo C. Intensified pharmaceutical care is improving immunosuppressive medication adherence in kidney transplant recipients during the first post-transplant year: a quasi-experimental study. Nephrol Dial Transplant. 2014;29(8):1597-607.

12. Maldonado AQ, Weeks DL, Bitterman AN, McCleary JA, Seiger TC, Carson RW, et al. Changing transplant recipient education and inpatient transplant pharmacy practices: a single-center perspective. Am J Health Syst Pharm. 2013;70(10):900-4.

13. Musgrave CR, Pilch NA, Taber DJ, Meadows HB, McGillicuddy JW, Chavin $\mathrm{KD}$, et al. Improving transplant patient safety through pharmacist discharge medication reconciliation. Am J Transplant. 2013;13(3):796-801.

14. Harrison JJ, Wang J, Cervenko J, Jackson L, Munyal D, Hamandi B, et al. Pilot study of a pharmaceutical care intervention in an outpatient lung transplant clinic. Clin Transplant. 2012;26(2):E149-57.

15. Chisholm MA, Mulloy LL, Jagadeesan M, DiPiro JT. Impact of clinical pharmacy services on renal transplant patients' compliance with immunosuppressive medications. Clin Transplant. 2001;15(5):330-6.

16. Pinelli NR, Clark LM, Carrington AC, Carrington JL, Malinzak L, Patel A. Pharmacist managed diabetes and cardiovascular risk reduction clinic in kidney transplant recipients: bridging the gap in care transition. Diabetes Res Clin Pract. 2014;106(3):e64-7.

17. Chisholm-Burns MA, Spivey CA, Graff Zivin J, Lee JK, Sredzinski E, Tolley EA. Improving outcomes of renal transplant recipients with behavioral adherence contracts: a randomized controlled trial. Am J Transplant. 2013; 13(9):2364-73.

18. Klein A, Otto G, Krämer I. Impact of a pharmaceutical care program on liver transplant patients' compliance with immunosuppressive medication: a prospective, randomized, controlled trial using electronic monitoring. Transplantation. 2009;87(6):839-47.

19. Chisholm MA, Spivey CA, Mulloy LL. Effects of a medication assistance program with medication therapy management on the health of renal transplant recipients. Am J Health Syst Pharm. 2007;64(14):1506-12.

20. Tschida S, Aslam S, Khan TT, Sahli B, Shrank WH, Lal LS. Managing specialty medication services through a specialty pharmacy program: the case of oral renal transplant immunosuppressant medications. J Manag Care Pharm. 2013;19(1):26-41. 
21. Chisholm MA, Mulloy LL, Jagadeesan M, Martin BC, DiPiro JT. Effect of clinical pharmacy services on the blood pressure of African-American renal transplant patients. Ethn Dis. 2002;12(3):392-7.

22. Partovi N, Chan W, Nimmo CR. Evaluation of a patient education program for solid organ transplant patients. Can J Hosp Pharm. 1995;48(2):72-8.

23. Impact pharmacie [website]. Montréal (QC): Centre hospitalier universitaire Sainte-Justine, Unité de recherche en pratique pharmaceutique; [cited 2018 Oct 4]. Available from: http://impactpharmacie.org/index.php?lang=01

24. Required educational outcomes, goals, and objectives for Postgraduate Year Two (PGY2) pharmacy residencies in solid organ transplant. Bethesda (MD): American Society of Health-System Pharmacists; [cited 2018 May 17]. Available from: https://www.ashp.org/-/media/assets/professionaldevelopment/residencies/docs/pgy2-solid-organ-transplant-pharmacy.ashx?la =en\&hash=52CA32882E85E9A431FFD9C2B34ACA09DB3F0A39

25. Chisholm MA. A renal transplantation advanced pharmacy practice experience. Am J Pharm Educ. 2006;15;70(1):3.

26. Renaudin P, Boyer L, Esteve MA, Bertault-Peres P, Auquier P, Honore S. Do pharmacist-led medication reviews in hospitals help reduce hospital readmissions? A systematic review and meta-analysis. Br J Clin Pharmacol. 2016;82(6):1660-73.

27. Transplant pharmacy community of practice (TxPharm COP). Mt Laurel (NJ): American Society for Transplantation; [cited 2018 May 25]. Available from: https://www.myast.org/communities-practice/txpharmcop

28. Practice and research networks: Immunology/transplantation PRN. Lenexa (KS): American College of Clinical Pharmacy; [cited 2016 Dec 14]. Available from: https://www.accp.com/about/prns.aspx

29. Board of Pharmacy Specialties receives petition to recognize solid organ transplantation pharmacy as a specialty. Washington (DC): Board of Pharmacy Specialties; 2018 Apr 4 [cited 2018 May 15]. Available from: https://www.bpsweb.org/2018/04/04/board-of-pharmacy-specialtiesreceives-petition-to-recognize-solid-organ-transplantation-pharmacy-as-aspecialty/

30. Pharmacist group. Ottawa (ON): Canadian Society of Transplantation; [cited 2018 May 24]. Available from: http://www.cst-transplant.ca/pharmacistgroup.html
31. PSN communities: Transplant. Ottawa $(\mathrm{ON})$ : Canadian Society of Hospital Pharmacists; [cited 2018 May 17]. Available from: https://www.cshp.ca/ psn-communities

Sébastien Sam, PharmD, is with the Pharmacy Practice Research Unit, Pharmacy Department, Centre hospitalier universitaire Sainte-Justine, Montréal, Quebec.

Aurélie Guérin, PharmD, is with the Pharmacy Practice Research Unit, Pharmacy Department, Centre hospitalier universitaire Sainte-Justine, Montréal, Quebec.

André Rieutord, PharmD, PhD, is with the Pharmacy Department, Hôpital Antoine-Béclère, Clamart, France.

Stéphanie Belaiche, PharmD, is with the Pharmacy Department, Centre hospitalier universitaire Lille, Lille, France.

Jean-François Bussières, BPharm, MSc, MBA, FCSHP, is with the Pharmacy Practice Research Unit, Pharmacy Department, Centre hospitalier universitaire Sainte-Justine, and the Faculty of Pharmacy, Université de Montréal, Montréal, Quebec

Competing interests: None declared.

Address correspondence to:

Jean-François Bussières

Pharmacy Department

Centre hospitalier universitaire Sainte-Justine

3175, chemin de la Côte Sainte-Catherine

Montréal QC H3T 1 C5

e-mail: jf.bussieres@ssss.gouv.qc.ca

Funding: None received.

\section{ON THE FRONT COVER}

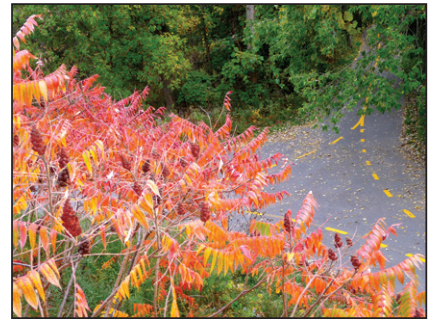

Near the North Branch of the Thames River London, Ontario

The cover photograph was taken by pharmacist Linda Hooper along the bike path that parallels the north branch of the Thames River in London, Ontario, near her workplace (University Hospital, London Health Sciences Centre). The camera was a Canon EOS 40D.

Linda commented that the scene brought to mind a poem by Canadian poet William Wilfred Campbell. "When I was a kid, this Canadian poem was our memory work at school."

The CJHP would be pleased to consider photographs featuring Canadian scenery taken by CSHP members for use on the front cover of the Journal. If you would like to submit a photograph, please send an electronic copy (minimum resolution $300 \mathrm{dpi}$ ) to publications@cshp.ca.
Indian Summer

Along the line of smoky hills

The crimson forest stands,

And all the day the blue-jay calls

Throughout the autumn lands.

Now by the brook the maple leans

With all his glory spread,

And all the sumachs on the hills

Have turned their green to red.

Now by great marshes wrapt in mist,

Or past some river's mouth,

Throughout the long, still autumn day

Wild birds are flying south.

- William Wilfred Campbell (1858?-1918) 